\title{
Structural Characterization of Monoclonal Antibody Therapeutics by Transmission Electron Microscopy and 2D Classification Analysis
}

\author{
Anette Schneemann ${ }^{1}$, Joyce J. Sung ${ }^{1}$, Jeffrey A. Speir ${ }^{1}$, Sean K. Mulligan ${ }^{1}$, Kat On ${ }^{1}$, Joel Quispe ${ }^{1}$, \\ Bridget Carragher ${ }^{1}$ and Clinton S. Potter ${ }^{1}$ \\ 1. NanoImaging Services, Inc., San Diego, CA USA
}

Monoclonal antibody (mAb) therapeutics represent the fastest growing sector in the biopharmaceutical industry. Significant advancement in immunoglobulin design has allowed synthesis of mAbs with increased binding affinity, improved conformational stability, high solubility and optimized effector functions. Of key interest are novel architectural $\mathrm{mAb}$ designs with enhanced bioactivity, particularly bi-specific antigen binding. A great variety of genetic engineering approaches are used to generate these novel types of mAbs and their structural features are largely unknown. Relative orientations of multiple variable domains, their conformational flexibility, and their accessibility to target antigens need to be evaluated on an individual basis. Crystallization and structure determination by X-ray analysis is difficult given the flexibility and conformational heterogeneity of these proteins. In contrast, transmission electron microscopy (TEM) represents a convenient and rapid approach to gain key information on structural and functional features of these types of molecules.

TEM is readily capable of providing two dimensional (2D) projection images of negatively stained antibody molecules. The domain structure of individual molecules can often be discerned at high magnification, but single projection images are noisy. The signal to noise ratio is significantly improved by averaging hundreds to thousands of individual antibody images, after classifying the molecules into identical spatial orientation. These $2 \mathrm{D}$ class averages reveal features not usually apparent in projection images of single molecules. They allow identification and location of genetically engineered domains, their relative disposition and the presence of bound antigens. Flexibility of the molecule can be assessed by measuring angles between known domains in different 2D class averages. In addition, movies generated from the 2D class averages after additional focused classification analysis effectively illustrate domain movements in 2D space. Finally, three-dimensional (3D) maps at moderate resolution can be reconstructed using the random conical tilt method.

We will demonstrate the power of these approaches in several examples. (i) TEM and 2D classification of chicken IgY revealed that these molecules are highly flexible and comparable to IgG in this regard despite the lack of a canonical hinge region. This result was surprising given current hypotheses which predict $\operatorname{IgY}$ to be a relatively rigid molecule. (ii) Analysis of turtle IgY revealed the existence of unconventional immunoglobulin domain structures, with one class of antibodies having a truncated Fc arm. (iii) Alignment and 2D class averaging of mammalian IgM, combined with selective masking and sub-classification, showed that the five antibody monomers are arranged asymmetrically around a central hub. This is in contrast to prevailing models, which show the monomers to be symmetrically arranged. (iv) The architecture and dynamics of a dual variable domain IgG (DVD-Ig) molecule and its parental $\mathrm{mAb}$ were examined. Hinge angles measured for the DVD-Ig molecule were similar to the inner antigen parental mAb. The outer binding domain of the DVD-Ig molecule was highly mobile and $3 \mathrm{D}$ analysis showed binding of inner antigen caused the outer domain to fold out of the plane with a major morphological change. Docking high-resolution X-ray structures into the 3D electron microscopy map further clarified the extraordinary domain flexibility observed in the DVD-Ig molecule allowing antigen binding with minimal steric hindrance. Together, these results demonstrate that TEM is well suited to address a range of issues in antibody structure and function that may be refractory to analysis by other experimental methods. 


\section{References:}

[1] Sung JJ et al, (2015). J Pharm Sci 104:750-9.

[2] Work TM et al, (2015). . J Immunol 195:5452-60.

[3] Correia I, et al. (2013). MAbs. 5:364-72.
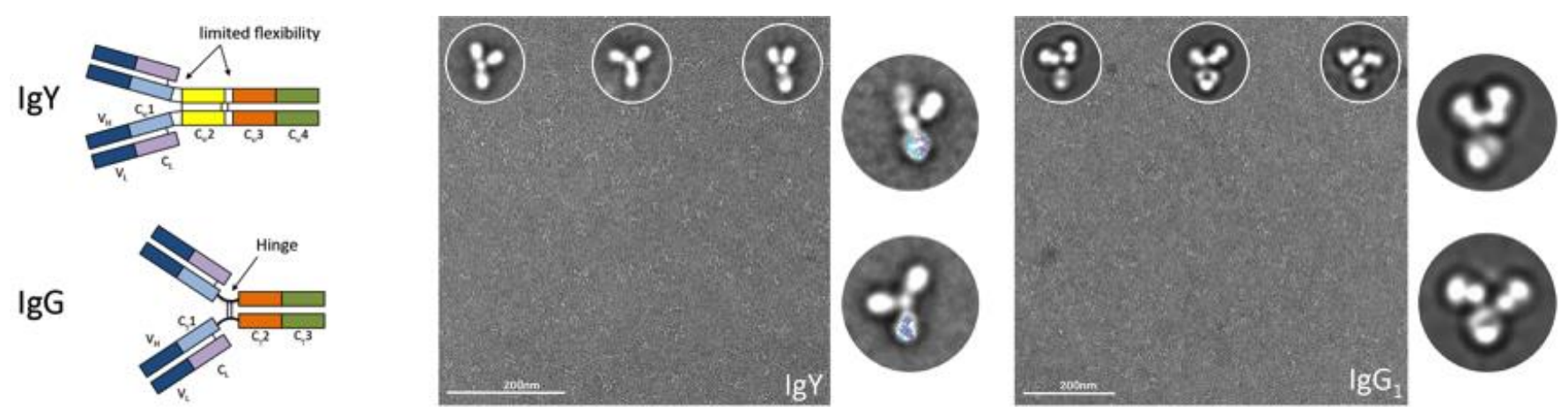

Figure 1. Structural characterization and flexibility analysis of chicken IgY. Left: Domain structure of avian $\operatorname{IgY}$ and mammalian IgG. The heavy chain of IgG contains three constant domains, C $\gamma 1-3$. C $\gamma 1$ is separated from $C \gamma 2$ by a hinge region, which confers considerable flexibility to the $\mathrm{F}_{\mathrm{ab}}$ arms. In contrast, the heavy chain of $\operatorname{IgY}$ contains four constant domains, Cu1-4. The Cu3 and Cu4 domains are closely related to the $\mathrm{C} \gamma 2$ and $\mathrm{C} \gamma 3$ domains of $\mathrm{IgG}$. The Cu2 domain is thought to have evolved into the hinge region in IgG. Middle and Right: TEM of chicken IgY and human $\mathrm{IgG}_{1}$. Insets and side bars show $2 \mathrm{D}$ class averages formed by aligning and averaging many particles. Both antibodies exist in a variety of conformers suggesting considerable flexibility of the molecules.
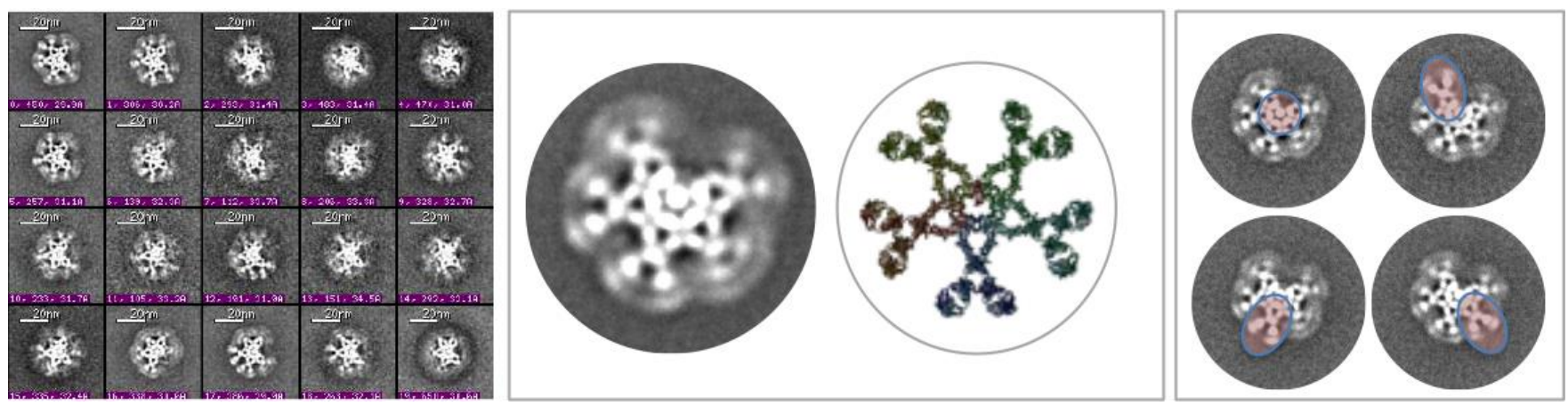

Figure 2. Alignment and classification of IgM. Left: Class averages of IgM. Middle: Representative class average of IgM shows five arms arranged asymmetrically. Model of IgM from SAXS and molecular graphics modeling (PDB ID: 2RCJ) shows symmetric arrangement of arms. Right: Selective masking (tinted region) and subsequent sub-classification support the asymmetrical arrangement of the IgM arms.

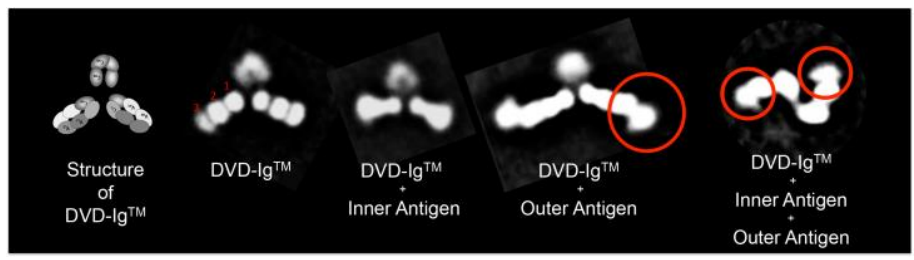

Figure 3. Characterization of dual variable domain immunoglobulin. Individual particles were selected from the images and aligned to form class averages of the DVD-Ig ${ }^{\mathrm{TM}}$ alone and bound to its antigens. Domain structure and antigen binding sites are visible. 